\title{
The Process of Coagulation in Smoke*
}

$I^{N}$ contradistinction to hydrosols, smokes are unstable systems. Their particles cohere when brought together by Brownian agitation, and this process of coagulation proceeds spontaneously until the system becomes a coarse suspension of complex aggregates, and finally sediments rapidly.

In recent years, special methods have been developed for counting the number of particles in many types of smoke, and the process of coagulation has been studied quantitatively in a variety of systems. It has been found experimentally that the decrease with time of the number of particles in a smoke is given to a first approximation by the expression $1 / n-1 / n_{0}=K t$, where $n$ is the number of particles present in a given volume at time $t, n_{0}$ the initial number and $K$ a constant. As is well known, an expression of the same form is valid for the recombination of ions, but in a normal smoke the combination of particles to form aggregates is only influenced slightly, if at all, by electrical charges.

A comparison of the coagulation constants or $K$ values for different smokes of about the same mass concentration shows that they vary between comparatively narrow limits from about $0.8 \times 10^{-9} \mathrm{~cm}^{3}$ sec. $^{-1}$ in the case of oxide smokes formed in the electric arc to $0.50 \times 10^{-9} \mathrm{~cm}^{3} \mathrm{sec}^{-1}$ for a standard smoke of stearic acid of mass concentration of $15 \mathrm{mgm}$. per cubic metre.

The structure of the aggregates and the nature of the material do not, so far as is known at present, exert any marked effect on the rate of coagulation, which appears to be a purely physical process dependent on the chance encounter of particles in Brownian motion. Experimental evidence, however, shows that the coagulation constant increases rapidly when the average size of particle falls below $1 \times 10^{-5} \mathrm{~cm}$. radius, and it increases also with the degree of heterogeneity of the smoke. By a careful study of the conditions under which a smoke is formed, Patterson and Cawood have prepared disperse systems of stearic acid particles which initially approach to uniformity in size. This was accomplished by dispersing the heated solid in a rapid

* Substance of the Liversidge Lecture delivered by Prof. $R$. Whytlaw-Gray, O.B.E., F.R.S., before the Chemical Society on February 14. blast of hot air, and by this means diluting rapidly the concentrated smoke before coagulation had proceeded far. Such smokes are readily reproducible and form standard systems which coagulate at the same rate and contain the same number of particles, and since they form compact aggregates they approximate in character to ideal systems of spherical particles.

The well-known theory of von Smoluchowski, which has been confirmed experimentally for the coagulation of sols by the comprehensive researches of Tuorila, when modified so as to apply to aerial systems, enables the coagulation constant of a homogeneous smoke to be calculated from first principles. Patterson and Cawood have shown that when the experimental data for these 'blown smokes' are interpreted rightly, a remarkably close agreement between theory and experiment is obtained. Theory also indicates that whilst in sols undergoing quick coagulation the rate should be independent of size, in aerial systems it should increase as the particle size diminishes; a prediction in entire conformity with experiment.

Both in sols and aerosols, theory shows that heterogeneity must increase the chances of encounter between particles, but although in sols it has been possible to check experimentally the extension of von Smoluchowski's theory proposed by H. Müller, for heterogeneous smokes theory so far has not proved more than a qualitative guide.

The study then of smokes affords strong confirmatory evidence of the validity of Smoluchowski's theory, and lends support to the view that this continuous process so characteristic of these systems is akin to the quick coagulation of sols in the presence of electrolytes, and points to the probability that in both classes of system every collision between particles is effective. It must, however, be noted that the coagulation constants for systems of fine suspensions in water and in air are widely different, the latter being about a hundred times as great as the former. But since the time taken to reduce the original number of particles in a system to any given fraction depends on the number as well as on $K$, the disappearance of particles by coagulation in town fogs and other polluted atmospheres will be slow.

\section{British Industries Fair, I935}

$\mathrm{I}^{\mathrm{N}}$ the account given last year in NATURE of the 1934 British Industries Fair, it was described as the largest national trade fair in the world. The 1935 Fair, which opened in London on February 18, is even larger than its immediate predecessor-a reflection, it may be hoped, of increasing prosperity in the nation's trade. At Olympia the lighter industries occupy every available square foot of exhibiting space, while the textile and furnishing sections at the White City are larger and more fully representative than ever. The main object of the Fair is, of course, a commercial one ; the most welcome visitors are buyers. But the Fair has, undoubtedly, an educational value, for it presents to the visitor, in an attractive and accessible form and in a condensed space, a general survey of the results of the nation's manufacturing industry. Moreover, every changing phase in the tastes and habits of the people is reflected in such a collection of manufactured articles as is to be found at the Fair. The removal of much that is tedious and unnecessary from domestic work is indicated by the increased popularity of chromiumplated ware and of stainless steel and stainless silver articles, and by the space allotted in the Fair to the exhibition of devices for domestic mechanisation. The exhibition in larger quantities of open-air equipment of all kinds indicates that increasingly the leisure of the people is being used in healthy pursuits. At Olympia, the exhibition is, as previously, divided into sections according to industries, and it is perhaps symbolic of the increasing recognition of the co-operation which must exist between science and industry, that the section devoted to the exhibition of the products of the Scientific and Optical 
Instruments Group should occupy a prominent position near the main entrance to the Fair. The exhibits of the various firms in this section are placed so compactly together that a visual impression is conveyed of the co-operation and joint effort which is to be found among the members of the industry. The instruments shown are mostly optica] in character, and one is reminded again of the large part played in scientific and industrial life by the products of the optical firms. Besides the normal instruments for laboratory equipment, special instruments are shown for use in aeronautics, astronomy and meteorology, together with those specially adapted to nautical and surveying requirements. One is impressed by the success which has been attained in so many of the instruments in combining the robustness required for industrial use with the necessary delicacy of movement.

Few things have been more striking of recent years than the development of long-distance telegraphy. It is but a short time ago that the first photographs were telegraphed from Australia to England, and quite recently a cinematograph film was exhibited in England showing events which had taken place but a few hours earlier in Australia. The exhibit of Cable and Wireless Ltd. is, on this account, of special interest. The modern system of long-distance telegraphy is admirably shown at its stand. The types of apparatus used for this work are presented in actual operation, and a study of the receiving and transmission units, magnifying relays, distortion removers and regenerators gives the visitor a clear idea of the inventive research which has enabled long-distance telegraphy to become part of his daily life. Realism is added to the demonstration by the fact that a written telegram, handed in at one end of the stand, is delivered as an automatically typed message at the other end after having passed through the complete system.

The chemical industry provides another example of the co-operation which may exist between firms in the same industry to their mutual benefit. Messrs. Hopkin and Williams and British Drug Houses Ltd. have combined their knowledge and experience to further the production of chemical reagents of an exceptionally high standard of purity, and these reagents are being exhibited by the firms. The very large field covered by the activities of Imperial Chemical Industries Ltd. is well illustrated by the fact that this firm has thought it worth while to devote a large portion of its space at the Fair, not to the display of its products, but to a presentation of its sales machinery. By means of interesting maps, the way in which chemical products enter into almost every phase of industry throughout Great Britain is clearly demonstrated. The same firm is making a special point of the hydrocyanic acid method of fumigation, especially for the cleansing of vermininfested houses. Great success is claimed for this method of fumigation, and specimens of the fumigant and of its prospective victims are shown. The same fumigant has a variety of other and more pleasant uses, the removal of fruit-damaging insect pests from orange groves being specially emphasised as being of interest to overseas visitors.

Of the other industries represented at the Fair at Olympia, no section is more attractive, and certainly none more colourful, than that of the glass and pottery trades. A more perfect blending of utility and beauty can scarcely be imagined. It is perhaps fitting that the products of possibly the most ancient craft represented at the Fair should appear so near to perfection. The beauty of the exhibits is enhanced by the excellent lighting, and the stands furnish an example of the use of modern lighting effects to improve the display of goods. Quite near to the glass and pottery section, on the floor above, is a large area devoted to plasties, and it is not uninteresting to compare the products of this very modern industry with those of the very ancient one mentioned above. The increasing use which is being made of plastic materials is reflected in the very large increase of space occupied by the plastic group. In this industry the gap between laboratory experiment and workshop practice has been most effectively and rapidly bridged.

The fact that in London alone more than fifteen hundred firms are exhibiting almost every variety of manufactured articles makes it obviously impossible in a limited space adequately to describe such a Fair. In addition to these firms, one hundred and thirty-four inventors are displaying for the first time to the purchasing public the results of their ingenuity, which range from rubber contrivances for the prevention of housemaid's knee to a mechanical device for 'breaking-in' new pipes.

At the White City every phase of textile manufacture is represented; whilst the furniture section at the same place presents a wide field of interest to those concerned with the evolution and modern developments of furniture. The Fair remains open until March 1. The Engineering and Hardware Section opens in Birmingham on May 20.

\section{University and Educational Intelligence}

Cambridge.- - At the Congregation of the Regent House a grace will be submitted appointing Prof. G. H. F. Nuttall, Magdalene College, emeritus professor of biology, Prof. E. D. Adrian, Trinity College, Prof. R. C. Punnett, Gonville and Caius College, Balfour professor of genetics and Mr. C. ForsterCooper, Trinity Hall, University reader in vertebrate zoology, delegates from the University to the tercentenary of the National Museum of Natural History in Paris next June.

At Newnham College the Henry Sidgwick Memorial Lecture will be delivered on March 9 in the College Hall at 5 p.m. by Sir John Russell, director of the Rothamsted Experimental Station. The subject of the lecture is "The Impact of Science on the National Life".

THE thirteenth Unity History School will be held in Rome on April 15-22. The subject of the meeting will be "Science in the Modern World". On April 15, the inaugural lecture entitled "Science and Philosophy" will be delivered by Prof. F. Enriques, president of the School of the History of Science, University of Rome. Other lectures will be delivered by Mr. F. S. Marvin, director of the Unity History Schools, Prof. H. Dingle, Dr. W. A. Parr, Prof. E. Radl, Dr. C. H. Desch, Prof. C. Formichi, Dr. G. Sarton, and M. Lheritier. Several discussions have also been arranged. Further information can be obtained from Mrs. K. E. Innes, 29 High Oaks Road, Welwyn Garden City, Herts.

Vocatronal guidance service finds a valuable auxiliary in the Journal of Careers (monthly, 1s.). The December issue contains "The Prospect in 